\title{
Computer-Assisted Minimally Invasive Curettage and Reinforcement of Femoral Head Osteonecrosis with a Novel, Expandable Blade Tool
}

\author{
Tsuyoshi Koyama ${ }^{1}$, Nobuhiko Sugano ${ }^{2}$, Hidenobu Miki², Takashi Nishii ${ }^{2}$, \\ Yoshinobu Sato $^{3}$, Hideki Yoshikawa ${ }^{2}$, Shinichi Tamura ${ }^{3}$, and Takahiro Ochi ${ }^{1}$ \\ ${ }^{1}$ Division of Robotic Therapy, Osaka University Graduate School of Medicine, \\ 2-2 Yamadaoka, Suita, 565-0871 Osaka, Japan \\ koyama@cl-comp.med.osaka-u.ac.jp \\ ${ }^{2}$ Department of Orthopaedic Surgery, Osaka University Graduate School of Medicine, \\ 2-2 Yamadaoka, Suita, 565-0871 Osaka, Japan \\ ${ }^{3}$ Division of Interdisciplinary Image Analysis, Osaka University Graduate School of Medi- \\ cine, 2-2 Yamadaoka, Suita, 565-0871 Osaka, Japan
}

\begin{abstract}
For minimally invasive curettage of femoral head osteonecrosis, we have developed a novel expandable blade tool which can be introduced into the femoral head through the subtrochanteric route under navigation guidance. In this study, we evaluated the effectiveness and feasibility of this tool in comparison with the Cebotome, a conventional bone cutter. A target area in the femoral head of a Sawbone femur model was curetted with each tool through the subtrochanteric route under navigation guidance. The volume of the curetted necrotic lesion was significantly larger and the procedure time was significantly shorter with this tool than with the Cebotome. The compressive strength of the femoral head curetted with this tool and filled with hydroxyapatite blocks was comparable to that of the intact one. This expandable blade tool can be a suggestion for more effective and feasible curettage of necrotic lesions in femoral head osteonecrosis than conventional bone cutters.
\end{abstract}

\section{Introduction}

The treatment of femoral head osteonecrosis is still controversial. It has been reported that about $70-80 \%$ of hips with femoral head osteonecrosis show progression of collapse of the femoral head if they do not receive any surgical treatment[5] (Fig. 1). In order to halt progression of collapse and to accelerate reparative process of the necrotic lesions, various joint preserving procedures have so far been performed. Core decompression with or without bone grafting[3] is one of the popular procedures in early stages. This procedure is performed through the lateral subtrochanteric route to the proximal femur which is comparatively less invasive than other methods, but this procedure has a limitation in curetting a large lesion through a long narrow hole. It has been reported that $20-40 \%$ of hips treated with this procedure in early stages resulted in collapse and needed total hip arthroplasty[1,3,4]. On the other hand, the trapdoor 
procedure[2] enables to curette a large lesion effectively through a trapdoor made in the femoral head. However, this procedure is quite invasive because the hip may need to be dislocated in order to make a trapdoor on the femoral head.

We think that the collapse of the femoral head in femoral head osteonecrosis may be prevented if the necrotic lesions are curetted thoroughly and the deficit is filled with reinforcement materials such as hydroxyapatite ceramics, bone grafts or bone substitutes with sufficient mechanical strength. For minimally invasive curettage of necrotic lesions of femoral head osteonecrosis, we have developed a novel, expandable blade tool which can be introduced into the femoral head through the lateral subtrochanteric route under a surgical navigation system. In this study, we evaluated the effectiveness and feasibility of this tool in comparison with the Cebotome ${ }^{\circledR}$, a popular conventional bone cutter, with respect to curettage of target necrotic lesions supposed in a Sawbone ${ }^{\circledR}$ femoral head model. Moreover, we evaluated the compressive strength of the Sawbone femoral head after the inside part of the femoral head was curetted with this tool and filled with hydroxyapatite blocks as reinforcement material.
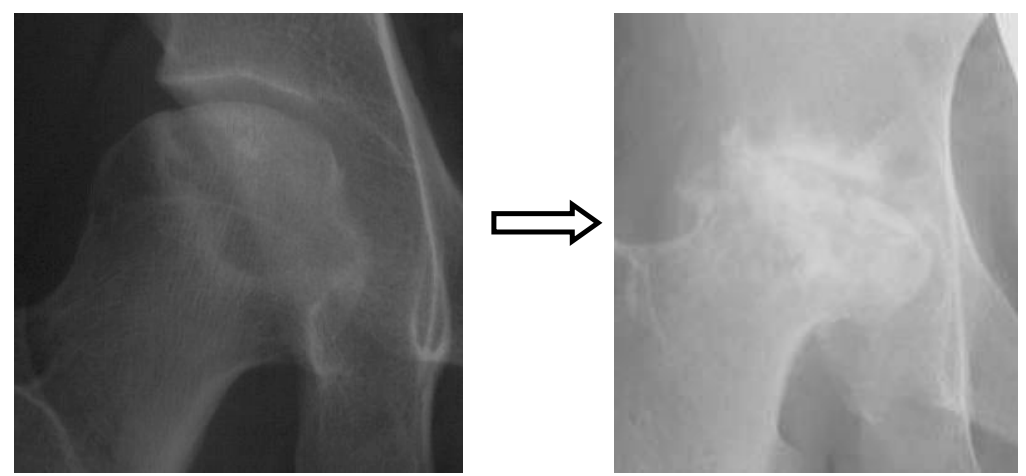

Fig. 1. Course of collapse in femoral head osteonecrosis

\section{Materials and Methods}

Our novel, expandable blade tool has a mechanism by which a $14-20 \mathrm{~mm}$ long metal blade housed in the tip of a long $10 \mathrm{~mm}$-diameter metal cylinder is made to protrude(Fig. 2 left). The length of the expanding blade is changeable according to the sizes of the femoral head and the necrotic lesions. The blade swings on a hinge and protrudes outwards from the cylinder to an angle of 60 degrees (Fig. 2 right) when a trigger attached to the tool is pulled. This tool is designed to be used after boring a hole into the femoral head from the lateral subtrochanteric portion of the femur with a drill of the same diameter (Fig. 3 B, C). As the cylinder spins at high speed, the blade protrudes gradually, cutting a cone shaped section of bone away. 

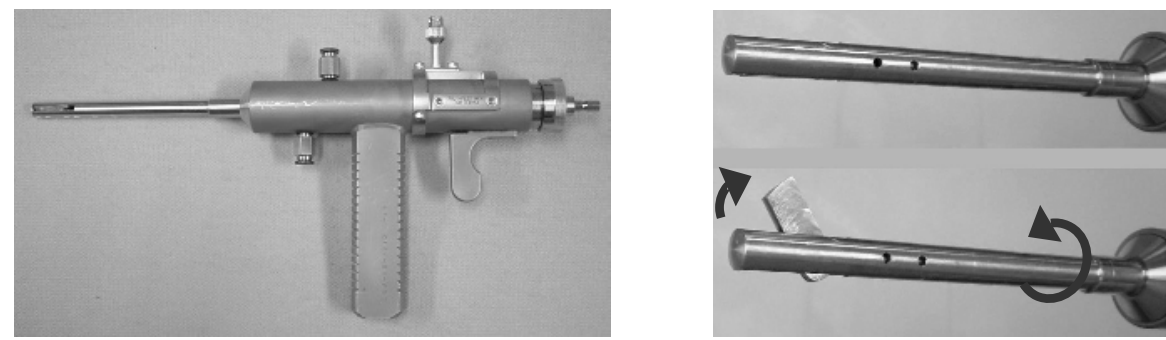

Fig. 2. The expandable blade tool
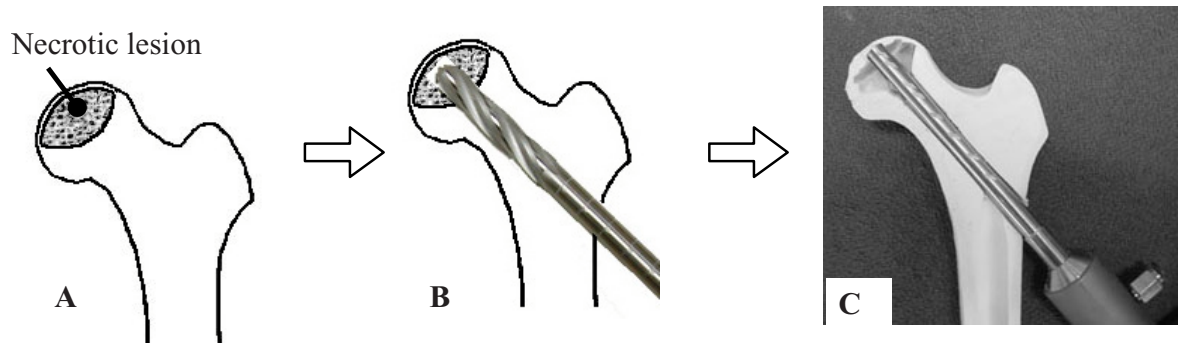

Fig. 3. Usage of the expandable blade tool

A: A typical extensive necrotic lesion of femoral head osteonecrosis

B: Coring a hole into the femoral head with a $10 \mathrm{~mm}$-diameter drill using the lateral subtrochanteric approach

C: Curettage of the necrotic lesion with the expandable blade tool

Study 1: The effectiveness and feasibility of the expandable blade tool were evaluated in comparison with the Cebotome ${ }^{\circledR}$ (MicroAire Surgical Instruments LLC, USA) (Fig. 4), a popular conventional bone cutter, using Sawbones ${ }^{\circledR}$ (Pacific Research Laboratories, USA) femur models.
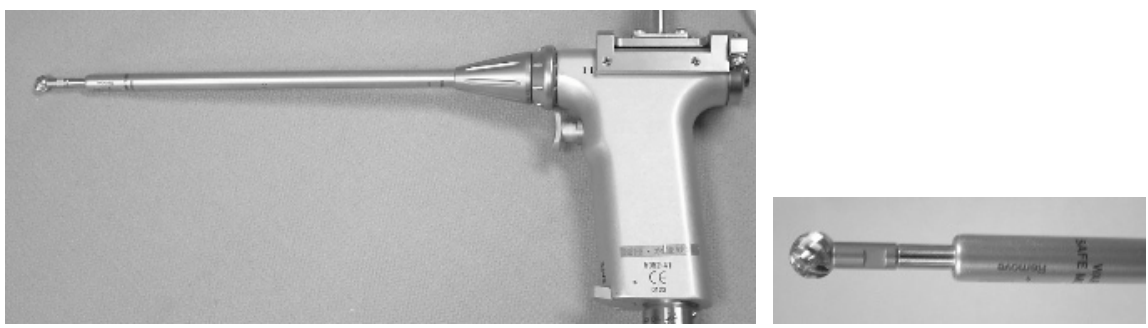

Fig. 4. Cebotome ${ }^{\circledR}$ 
A typical extensive necrotic lesion of femoral head osteonecrosis (type C2, by the Classification of the Japanese government investigation group[6]) was created virtually in the three-dimensional surface model of a Sawbone femur on the computer (Fig. 3A). A pre-operative planning to curette the target necrotic lesion with the expandable blade tool or with the Cebotome was carried out on the three-dimensional models on computer so that the target necrotic lesion was curetted maximally with each tool.

The whole procedure of curetting the Sawbone femoral head was performed under a CT-based optical surgical navigation system with an optical three-dimensional position sensor, OPTOTRAK ${ }^{\circledR} 3020$ system (Northern Digital Inc., Canada). Optical tracking markers were attached to the Sawbone femur and to the surgical tools. First, the surface registration of the Sawbone femur under an optical navigation system was performed. Then, a guide wire was inserted from the lateral subtrochanteric portion of the femur into the femoral head under the navigation guidance according to the preoperative planning. After drilling with a $10 \mathrm{~mm}$-diameter drill over the guide wire to within $4 \mathrm{~mm}$ of the articular surface of the femoral head, the inside of the femoral head was curetted with the expandable blade tool or with the Cebotome in line with the preoperative planning (Fig. 5).

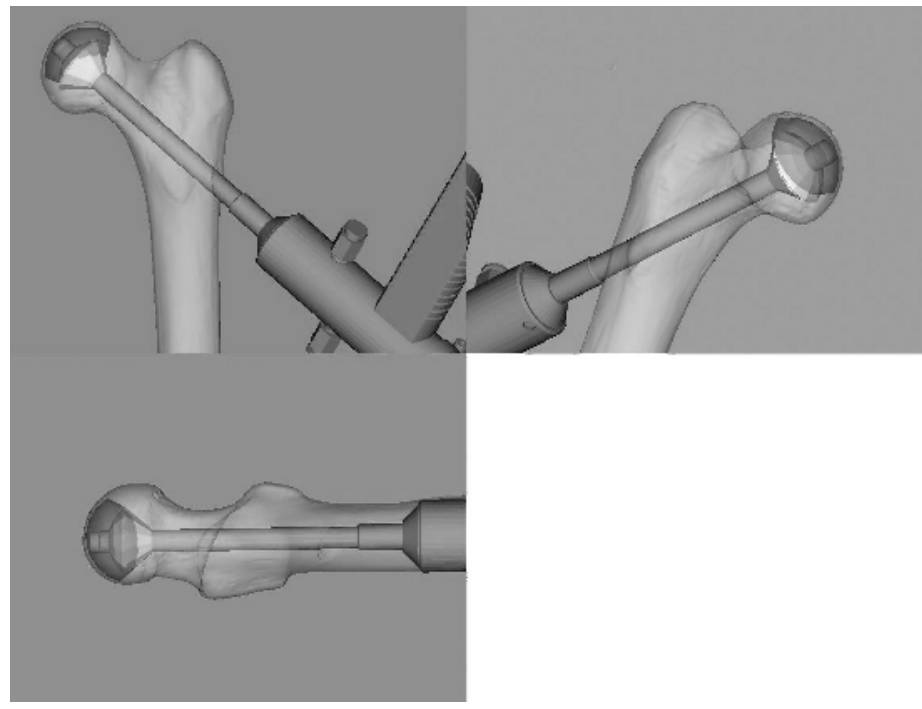

Fig. 5. Curetting the target necrotic lesion with the expandable blade tool under an optical navigation system.

After curettage, CT scans of the curetted Sawbone femur models were carried out. The CT image of the Sawbone femur after curettage was matched to the pre-operative planning image on the computer, and the volume of the actually curetted necrotic lesion and that of the sacrificed normal area of the femoral head were measured from the CT images.

The procedure time taken for the curettage after drilling a hole was also measured. The trials were repeated ten times for each surgical tool. Statistical analyses were performed using the Student's $t$-test with a significance level of 0.05 . 
Study 2: After curetting the Sawbone femoral head with the expandable blade tool in Study 1, the cavity in the femoral head was filled maximally with blocks of hydroxyapatite ceramics $3.3 \times 3.3 \times 5 \mathrm{~mm}$ in size (HA Block ${ }^{\circledR}$, Pentax, Japan) and compressive strength of the femoral head was examined. Compressive strength of the femoral head was measured at the weight-bearing portion of the femoral head with a servohydraulic strength testing system (ServoPulser ${ }^{\circledR}$, Shimadzu, Japan) (Fig. 6). Five specimens each were tested of: intact Sawbone femur models, models that had been curetted only, and models that had been curetted and filled with the hydroxyapatite blocks. Compression was produced with a $12.7 \mathrm{~mm}$-diameter cylindrical indentor attached to the testing machine.

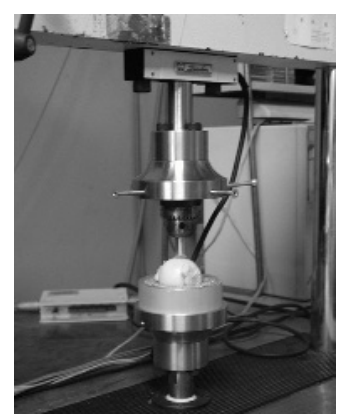

Fig. 6. Compressive strength of the femoral head was measured with a servohydraulic strength testing system.

\section{Results}

Study 1: The volumes of the curetted necrotic lesion and the volumes of the sacrificed normal area with each surgical tool are shown in Fig. 7.

The procedure time taken for the curettage with each surgical tool after drilling the hole is shown in Fig. 8.

Study 2: The compressive strength of the femoral head of the intact Sawbone femur models, models that had been curetted only, and models that had been curetted and filled with hydroxyapatite blocks are shown in Fig. 9.

\section{Discussion}

The volume of the curetted necrotic lesion was significantly larger and the procedure time was significantly shorter with the expandable blade tool than with the Cebotome. It can be stated that this expandable blade tool is both feasible and more effective than the Cebotome in curetting the necrotic lesions of femoral head osteonecrosis. The volume of the sacrificed normal area was significantly larger with the expandable blade tool than the Cebotome, however, this sacrificed area is near to the center of the 
femoral head where the bone mineral density is low, therefore the strength loss of the whole femoral head is supposed to be comparatively small. In fact, the compressive strength test showed that the femoral head that had been curetted with the expandable blade tool and filled with hydroxyapatite blocks had compressive strength comparable to that of the intact femoral head.

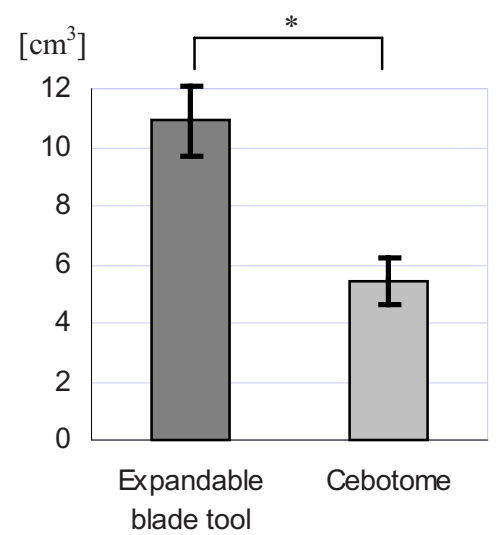

A: The volume of the curetted necrotic lesion

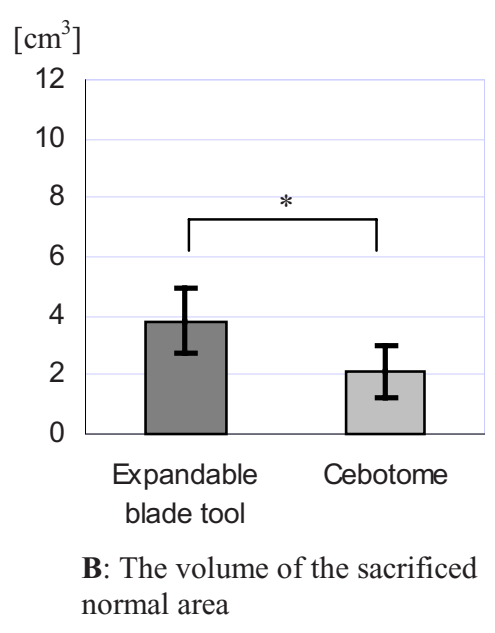

Fig. 7. The volumes of the curetted necrotic lesion (A) and the volumes of the sacrificed normal area in the femoral head $(\mathbf{B}) . \quad(* P<0.01)$

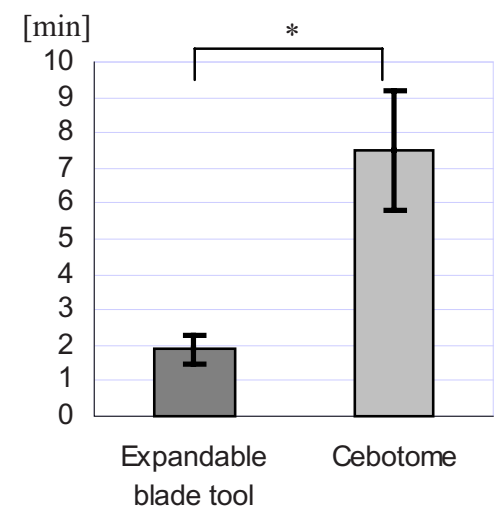

Fig. 8. The procedure time taken for the curettage after drilling the hole. $(* P<0.01)$

In terms of safety, with the expandable blade tool, only the bone on the side part of the tool can be cut away, therefore the risk of breaking through the surface of the femoral head is low. Moreover, because the diameter of the cylinder of this tool is the same as 
the drilled long hole, this tool remains stabilized when the cylinder is spinning at high speed.

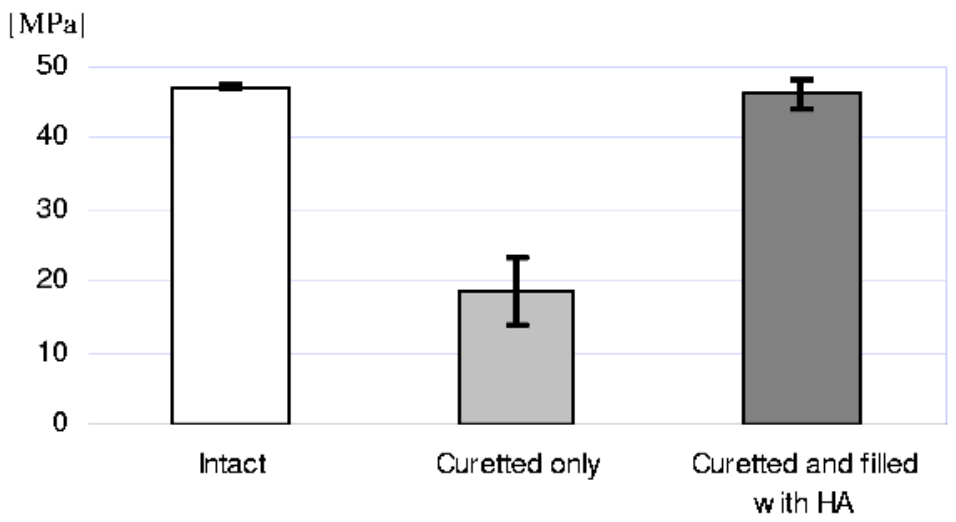

Fig. 9. The compressive strength of the femoral head.

\section{Conclusion}

Curettage of the necrotic lesion of femoral head osteonecrosis using the novel, expandable blade tool is both feasible and more effective than the Cebotome conventional bone cutter. The femoral head that has been curetted with this tool and filled with hydroxyapatite blocks has compressive strength comparative to that of the intact one. This surgical tool enables minimally invasive and effective curettage of the necrotic lesions in femoral head osteonecrosis.

\section{References}

1. Mont MA et al: Core decompression versus nonoperative management for osteonecrosis of the hip. Clin. Orthop. 324:169-178, 1996.

2. Mont MA et al: The trapdoor procedure using autogenous cortical and cancellous bone grafts for osteonecrosis of the femoral head. J. Bone Joint Surg. Br. 80(1):56-62, 1998.

3. Steinberg ME et al: Core decompression with bone grafting for osteonecrosis of the femoral head. Clin. Orthop. 386:71-78, 2001.

4. Stulberg BN et al: Osteonecrosis of the femoral head. A prospective randomized treatment protocol. Clin. Orthop. 268:140-151, 1991.

5. Sugano $\mathrm{N}$ et al: Prognostication of nontraumatic avascular necrosis of the femoral head. Significance of location and size of the necrotic lesion. Clin. Orthop. 303:155-164, 1994.

6. Sugano N, Atsumi T, Ohzono K, Kubo T, Hotokebuchi T, Takaoka K.: The 2001 revised criteria for diagnosis, classification, and staging of idiopathic osteonecrosis of the femoral head. J. Orthop. Sci. 7(5):601-605, 2002. 